\title{
A review on various brain tumor detection techniques in brain MRI images
}

\author{
Komal Sharma ${ }^{1}$, Akwinder Kaur ${ }^{2}$, Shruti Gujral ${ }^{3}$ \\ Research Scholar ${ }^{1}$, Assistant Professor ${ }^{2}$, Assistant Professor ${ }^{3}$ \\ Department of Computer Science, Chandigarh University, Gharuan, Mohali, India
}

\begin{abstract}
Magnetic resonance imaging is important imaging technique used in the detection of brain tumor. Brain tumor is one of the most dangerous diseases occurring among the human beings. Brain MRI plays a very important role for radiologists to diagnose and treat brain tumor patients. Study of the medical image by the radiologist is a time consuming process and also the accuracy depends upon their experience. Thus, the computer aided systems becomes very necessary as they overcome these limitations. Several automated methods are available, but automating this process is very difficult because of different appearance of the tumor among the different patients. There are various feature extraction and classification methods which are used for detection of brain tumor from MRI images. In this paper, various approaches are reviewed enlightening the advantages and disadvantages of these methods.
\end{abstract}

Keywords: - Feature extraction, Image segmentation, Clustering, Neural Network, MRI image, Tumor detection

\section{INTRODUCTION}

MRI is a fairly new technique that has been used by radiologist to envision the structure and function of the human body. The MRI scanner uses magnetic fields and the radio waves to create images of various tissues, organs and some other structures within the body. MRI instrument uses three electromagnetic fields - i) Static field which is very strong static magnetic field which is used to polarize the hydrogen nuclei of water molecules, ii) Gradient field which is weaker time varying field used for spatial encoding, iii) Weak radio frequency field which is used for manipulation of hydrogen nuclei which produce measurable signals collected through Radio Frequency antenna.MRI of the brain can be obtained to obtain the changes concerned with bleeding and also when the brain has been starved of oxygen after a stroke. It can be used to characterize tumors (benign or malignant) and check their progression.

MRI imaging technique is very useful in early detection of abnormalities in the brain. It is based on nuclear magnetic resonance phenomenon. MRI uses an effective magnetic field to line up the nuclear magnetization of hydrogen atoms in water in the body of the human beings.

The human body is consisting of various types of cells and every cell has its own specific function. On losing the ability to grow properly, cells start growing in an improper fashion and in an unordered manner. Extra cells are grouped together to form a mass of tissue which is called tumor. These cells can be categorized in two ways: Benign and malignant. Benign tumors do not contain cancer cells and malignant tumors contain tumor cells.

Various researches and studies have been carried out on both type of tumors till date. These studies show that rate of tumors in human body has been increasing and there is no clue of their such a fast rate of increasing. Medical imaging techniques and analysis tools which play a vital role in the field of clinical study also enable the doctors and radiologists to arrive at a specific diagnosis.

The examination involving MRI brain classification on the MRI images is also required. The aim of classification is to group items which have similar feature values into categories. A number of studies has been done on brain MRI classification. The classification is done using techniques such as neural networks, fuzzy cmeans, k-nearest neighbors and Support Vector Machine (SVM) as shown in figure 1.

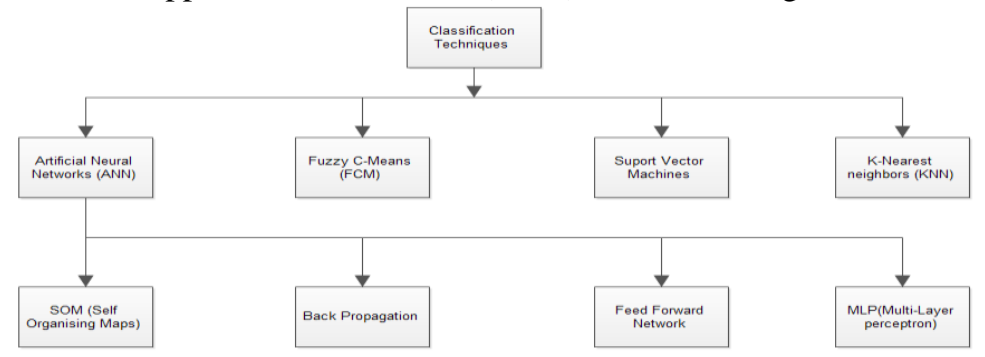

Fig 1. Classification Techniques 
The MR images data is by nature are very complex . Accurate diagnosis of MR images data is not an easy task and is always time consuming. In some extreme scenario, diagnosis with wrong result and delay in delivery of a correct diagnosis decision could occur due to the complexity and cognitive process of which it is involve.

The diagnosis of magnetic resonance human brain images is possible via supervised techniques such as artificial neural networks and support vector machine (SVM), and unsupervised classification techniques unsupervised such as self-organization map (SOM) and fuzzy c-means combined with feature extraction techniques like texture feature extraction, intensity feature extraction. Some other supervised classification techniques such as k-nearest neighbors (k-NN) also group pixels based on their similarities in each feature image can be used to classify the normal/pathological T2-wieghted MRI images.

Brain tumour can occur in different parts of the brain. The most common brain tumours are Gliomas, Oligodendroglioma ,Meningiomas, Glioblastoma, Pituitary adenomas and Nerve sheath tumours. Detection and segmentation of brain tumour- in MR images is very important in medical diagnosis. Brain tumor is the most dangerous diseases occurring commonly among the human beings, so study of brain tumor is very important.

\section{OVERVIEW}

Natarajan et al. [1] proposed brain tumor detection method for MRI brain images. The MRI brain images are first pre-processed using median filter, then segmentation of image is done using threshold segmentation and morphological operations are applied and then finally, the tumor region is obtained using image subtraction technique. This approach gives the exact shape of tumor in MRI brain image. Joshi et al. [2] proposed brain tumor detection and classification system in MR images by first extracting the tumor portion from brain image, then extracting the texture features of the detected tumor using Gray Level Co-occurrence Matrix (GLCM) and then classified using neuro-fuzzy classifier. Amin and Mageed [3] proposed neural network and segmentation base system to automatically detect the tumor in brain MRI images. The Principal Component Analysis (PCA) is used for feature extraction and then Multi-Layer Perceptron (MLP) is used classify the extracted features of MRI brain image. The average recognition rate is $88.2 \%$ and peak recognition rate is 96.7\%. Sapra et al. [4] proposed image segmentation technique to detect brain tumor from MRI images and then Probabilistic Neural Network (PNN) is used for automated brain tumor classification in MRI scans. PNN system proposed handle the process of brain tumor classification more accurately. Suchita and Lalit [5] proposed unsupervised neural network learning technique for classification of brain MRI images. The MRI brain images are first pre-processed which include noise filtering, edge detection, then the tumor is extracted using segmentation. The texture features are extracted using Gray-Level Co-occurrence Matrix(GLCM) and then Self-Organizing Maps (SOM) are used to classify the brain as normal or abnormal brain, that is, whether it contain tumor or not. Rajeshwari and Sharmila [6] proposed pre-processing techniques which are used to improve the quality of MRI image before using it into an application. The average, median and wiener filters are used for noise removal and interpolation based Discrete Wavelet Transform (DWT) technique is used for resolution enhancement. The Peak Signal to Noise Ratio (PSNR) is used for evaluation of these techniques.

George and Karnan [7] proposed MRI image enhancement technique based on Histogram Equalization and Center Weighted Median (CWM) filter as they are used to enhance the MRI image more effectively. Daljit Singh et al. in [8] proposed a hybrid technique for automatic classification of MRI images by first extracting the features using Principal Component Analysis (PCA) and Gray-Level Co-occurrence Matrix(GLCM) and then extracted features are fed as an input to Support Vector Machine(SVM) classifier which classifies the brain image as normal or abnormal. Gadpayleand and Mahajani [9] proposed brain tumour detection and classification system. The tumor is extracted using segmentation and then texture features are extracted using GLCM and finally the BPNN and KNN classifiers are used to classify the MRI brain image into normal or abnormal brain. The accuracy is $70 \%$ using KNN classifier and $72.5 \%$ by using BPNN classifier. Shasidhar et al. in [10] proposed modified Fuzzy C-Means (FCM) algorithm for MR brain tumor detection. The texture features are extracted from brain MR image and then modified FCM algorithm is used for brain tumor detection. The average speed-ups of as much as 80 times a traditional FCM algorithm is obtained using the modified FCM algorithm. The modified FCM algorithm is a fast alternative to the traditional FCM technique.

\section{FEATURE EXTRACTION}

Large amount of information is needed to represent an image and this information occupies large amount of memory. The features are extracted from the image. The extracted features contains the relevant information about the image. The extracted features are used as input to the classifier for classification. The features extracted methods for an image are described below. 


\subsection{Texture Based Features:-}

The texture extraction means extracting the homogeneity of an image and similarity between regions of an image. Texture feature classifies MRI of brain helps in detection of tumor in brain MRI image. The various texture features are described below:-

\subsubsection{Energy:-}

It gives the measure of homogeneity. It is also known as angular second moment or uniformity.

$$
\mathrm{E}=\sum_{i=0}^{N g-1} \sum_{j=0}^{N g-1} p(i . j)^{2}
$$

\subsubsection{Contrast:-}

It gives the measure of the intensity variation between the reference pixel and the neighbouring pixel.

$$
\text { Contrast }=\sum_{n=0}^{N g-1} n^{2} \sum_{i=0}^{N g-1} \sum_{j=0}^{N g-1} p(i . j)^{2}
$$

\subsubsection{Correlation :-}

It gives the measure of the intensity variation between the reference pixel and neighborhood pixel.

Correlation $=\frac{1}{\sigma^{x} \sigma^{y}} \sum_{i-0}^{N g-1} \sum_{j=0}^{N g-1}(i, j) p(i, j)^{2}-\mu_{x} \mu_{y}$

\subsubsection{Sum of Squares (Variance) :-}

It gives the measure of the gray tone variance of an image.

Variance, $\sigma^{2}=\sum_{i=0}^{N g-1} \sum_{j=0}^{N g-1}(1-\mu)^{2} \cdot p(i, j)$

\subsubsection{Inverse Difference Moment (IDM):-}

It gives the measure of the local homogeneity of an image.

$\mathrm{IDM}=\sum_{i=0}^{N g-1} \sum_{j=0}^{N g-1} \frac{1}{1+(i-j)^{2}} \cdot p(i . j)$

3.1.6 Entropy :-

It gives the amount of information of an image which is used for image compression.

Entropy, $\mathrm{EN}=\sum_{i=0}^{N g-1} \sum_{j=0}^{N g-1} p(i . j) \log p(i, j)$

Advantages of Texture based features:-

- It shows relatively good discrimination between the tumor and its surrounding edema.

Disadvantages of Texture based features:-

- These methods cannot segment all components of the tumor.

- These features are very sensitive to noise and inhomogeneity.

\subsection{Intensity Based Features:-}

Intensity based features are most commonly used features used in the classification. The intensity based features include mean, median, mode, skewness, kurtosis, energy, entropy. Let $\mathrm{f}(\mathrm{x}, \mathrm{y})$ - a two dimensional image, $\mathrm{h}(\mathrm{i})$ - the intensity level of an image, $\mathrm{N}_{\mathrm{g}}$ - the total number of gray levels in the entire image and $\mathrm{p}(\mathrm{i})$ - the probability density.

\subsubsection{Mean:-}

The mean gives the average level intensity of an image.

$$
\text { Mean, } \mu=\sum_{i=0}^{N g-1} \text { i. p(i) }
$$




$$
\begin{aligned}
& \text { where } \mathrm{p}(\mathrm{i})=\frac{h(i)}{N_{x} N_{y}} \quad, \mathrm{i}=0,1,2, \ldots \ldots \ldots \ldots . ., \mathrm{N}-1 \\
& \mathrm{~h}(\mathrm{i})=\sum_{\mathrm{x}=0}^{\mathrm{Ng}-1} \sum_{\mathrm{y}=0}^{\mathrm{Ng}-1} \alpha(\mathrm{f}(\mathrm{x}, \mathrm{y}), \mathrm{i}), \mathrm{i}=0,1,2, \ldots \ldots \ldots \ldots ., \mathrm{N}-1
\end{aligned}
$$

\subsubsection{Variance:-}

The variance gives variation of the intensity around the mean.

$$
\text { Variance, } \sigma^{2}=\sum_{i=0}^{N g-1}(1-\mu)^{2} . p(i) \text {. }
$$

\subsubsection{Standard Deviation:-}

$$
\mathrm{SD}, \sigma=\sqrt{\sum_{i=0}^{N g-1}(1-\mu)^{2} \cdot p(i)} .
$$

\subsubsection{Skewness:-}

The skewness gives the symmetry of an image.

$$
\mu^{3}=\sigma^{-3} \sum_{i=0}^{N g-1}(1-\mu)^{3} \cdot p(i)
$$

\subsubsection{Energy:}

Energy gives the measure of sum of the squared elements.

$$
\text { Energy, } \mathrm{E}=\sum_{i=0}^{N g-1}(p(i))^{2}
$$

\subsubsection{Entropy:}

Entropy gives the measure of uncertainty in a random variable.

Entropy, En $=-\sum_{i=0}^{N g-1} p(i) \log _{2} p(i)$

\section{Advantages of Intensity based features:-}

- These features directly indicate the physical characteristics of the tissues in brain MRI.

- Identification of tumor is fast by the using physical characteristics.

\section{Disadvantages of Intensity based features:-}

- Different tissues in MRI may have overlapping intensity values.

- Other information like anatomic knowledge should also be considered for achieving good results.

\section{CLASSIFICATION TECHNIQUES}

There are various classification techniques used for classifying brain as normal or abnormal . These classification techniques are described below :-

\subsection{Artificial Neural Network :-}

In this technique, the image is mapped into a Neural Network. The neural network works in two phases- the training phase and the testing phase. Firstly the neural network was trained with training examples in the training phase. After training, the neural network is tested on the unknown instances. Neural network technique includes important step that is feature extraction. Feature extraction is very important as the features that are extracted forms the input part of the neural network. 


\section{Artificial Neural Network is divided into 2 categories:-}

1. Feed-Forward Neural Network.

2. Recurrent Network or Feed-Backward Network.

In feed-forward neural network, the neurons are arranged in layers and they have unidirectional connections between them. They produce only one set of output values. They are called as static network because in this the output values are produced only based on current input. The output values does not depend on previous input values. They are also called as memory less network. In feedback network, the neurons have bidirectional connections between them. Feedback or Recurrent networks produce a set of values which depends on the previous input values. Feedback network is also known as dynamic network because the output values always depend on the previous input values.

Back Propagation algorithm is used in feed-forward neural network. In this network, the neurons are arranged in layers and send the output in the forward direction. The errors generated are back propagated in the backward direction to the input layer. The network receives the input by neurons in the input layer of the neural network and the output of the network is given by the neurons on an output layer of the neural network. The neural network consists of one or more intermediate hidden layers. In back propagation algorithm, the supervised learning is used. The error between the input and the computed output is calculated and back propagated. The network is trained with random weights and then later the weights are adjusted by back propagation to get the minimal error. The network is perfect if the error is minimal. In back propagation, the weights are changed each time such that the error reduces gradually. This is repeated until there is no change in the error.

Advantages of artificial neural network :-

- The neural networks have high parallel ability and fast computing.

- Expert intervention is reduced during the whole process.

Disadvantages of artificial neural network :-

- Some of the information should be known beforehand.

- They should be first trained using learning process beforehand.

- Period of training neural networks may be very long.

\subsection{Fuzzy C-Means :-}

It is a method of clustering. In this method, one pixel may belong to two or more clusters which represents group. In this algorithm, the finite collection of pixels are partitioned into a group of "c" fuzzy clusters according to some given criterion. The objective function of this algorithm is defined as the sum of distances between cluster centers and patterns. Different types of similarity measures are used to identify classes depending on the data and the application in which it is to be used. Some examples which can be used as similarity measures are intensity distance and connectivity.

The algorithm contain following steps:-

- Initialize the matrix M.

- Centers vectors are calculated.

- Perform K steps until the termination value is reached.

Advantages of fuzzy c-means :-

- It is very simple and fast algorithm.

- This algorithm is more robust to noise and provides better segmentation quality.

\section{Disadvantages of fuzzy c-means :-}

- It considers only image intensity values.

\subsection{Support Vector Machines(SVM) :-}

SVM is a supervised classifier with associated learning algorithm. The SVM based on the training samples. It attempts to minimize the bound on the generalization error . The generalisation error is the error made by the learning machine on the test data not used during training phase. Thus, the SVM always performs well when applied to data which is outside the training set. SVM uses this advantage and focus on the training examples which are difficult to classify. These "borderline" training examples which are difficult to classify are called as support vectors. SVM formulation is somewhat modified by adding least squares term in its cost function. It helps to circumvent the need to solve a more difficult quadratic programming problem and only 
requires the solution of a set of linear equations. This approach significantly reduces the complexity and computation in solving the problem of classification. It is based on the hyperplane and the hyperplane maximizes the separating margin among the two classes.

Support Vector Machines (SVM) works in the two stages- the training stage and the testing stage. SVM trains itself by learning features which are given as input to its learning algorithm. During the training phase, SVM selects the suitable margins between its two classes. Artificial neural network has a number of issues like having local minima and selection of number of neurons for each problem. Thus, the SVM classifier has no local minima.

SVM is a systematic and effective method for two class problems. The MRI brain images are classified into two separate classes such as normal class and abnormal class using SVM classifier. The SVM classifier method is better than rule based systems.

\section{Advantages of support vector machines :-}

- This algorithm has high generalization performance.

- It works well in case of high dimensional feature space.

- This algorithm works independent of the dimensionality of the feature space.

- The results given by support vector machines are very accurate.

Disadvantages of support vector machines :-

- The training time is very long.

- This algorithm is highly dependent on the size of data.

\subsection{K-Nearest Neighbour (KNN) :-}

The KNN algorithm is based on a distance function (Euclidean Distance) and a voting function is used for the k-Nearest Neighbours. The distance metric used is the Euclidean distance, It shows the higher accuracy and stability for MRI images than other classifiers. The KNN algorithm has a slow running time. The segmentation steps of KNN algorithm are as following :-

- Determine $\mathrm{k}$ value where $\mathrm{k}$ gives the number of nearest neighbors.

- Distance between query instance and all the training samples is calculated.

- On the basis of kth minimum distance, the distance is sorted.

- The majority class is assigned.

- The class is determined.

- The brain abnormalities are segmented.

Advantages of KNN algorithm :-

- KNN algorithm is fairly simple to implement.

- Real time image segmentation is done using $\mathrm{KNN}$ algorithm as it runs more quickly.

\section{Disadvantages of KNN algorithm :-}

- There is some possibility of yielding an erroneous decision if the obtained single neighbour is an outlier of some other class.

\section{CONCLUSION}

In this paper, various methods and techniques that are being used to detect the brain tumor from MRI images of brain are reviewed. A method which performs well for one MRI brain image may not perform for the other MRI brain image. So, it is very hard to achieve a general method that can be used for all MRI brain images. The merits and demerits of various techniques for brain tumour detection are analyzed in this paper. Several hybrid approaches may be developed through the combination of these techniques.

\section{REFERENCES}

[1] Natarajan P, Krishnan.N, Natasha Sandeep Kenkre, Shraiya Nancy, Bhuvanesh Pratap Singh, "Tumor Detection using threshold operation in MRI Brain Images", IEEE International Conference on Computational Intelligence and Computing Research, 2012.

[2] Dipali M. Joshi, N. K. Rana, V. M. Misra, "Classification of Brain Cancer Using Artificial Neural Network" , IEEE International Conference on Electronic Computer Technology ,ICECT ,2010.

[3] Safaa E.Amin, M.A. Mageed," Brain Tumor Diagnosis Systems Based on Artificial Neural Networks and Segmentation Using MRI", IEEE International Conference on Informatics and Systems, INFOS 2012. 
[4] Pankaj Sapra, Rupinderpal Singh, Shivani Khurana, "Brain Tumor Detection Using Neural Network", International Journal of Science and Modern Engineering, IJISME ,ISSN: 2319-6386, Volume-1, Issue-9, August 2013.

[5] Suchita Goswami, Lalit Kumar P. Bhaiya, " Brain Tumor Detection Using Unsupervised Learning based Neural Network" , IEEE International Conference on Communication Systems and Network Technologies, 2013.

[6] S. Rajeshwari, T. Sree Sharmila, "Efficient Quality Analysis of MRI Image Using Preprocessing Techniques" ,IEEE Conference on Information and Communication Technologies, ICT 2013.

[7] E. Ben George, M.Karnan, "MRI Brain Image Enhancement Using Filtering Techniques", International Journal of Computer Science \& Engineering Technology ,IJCSET, 2012.

[8] Daljit Singh, Kamaljeet Kaur, "Classification of Abnormalities in Brain MRI Images Using GLCM, PCA and SVM", International Journal of Engineering and Advanced Technology (IJEAT) ISSN: 2249 - 8958, Volume-1, Issue-6, August 2012.

[9] Prachi Gadpayleand, P.S. Mahajani, "Detection and Classification of Brain Tumor in MRI Images ", International Journal of Emerging Trends in Electrical and Electronics, IJETEE - ISSN: 2320-9569, Vol. 5, Issue. 1, July-2013.

[10] M. Shasidhar , V.Sudheer Raja, B. Vijay Kumar, "MRI Brain Image Segmentation Using Modified Fuzzy C-Means Clustering Algorithm" ,IEEE International Conference on Communication Systems and Network Technologies, 2011.

[11] T. Rajesh, R. Suja Mani Malar," Rough Set Theory and Feed Forward Neural Network Based Brain Tumor Detection in Magnetic Resonance Images" ,IEEE International on Advanced Nanomaterials \& Emerging Engineering Technologies, 2013.

[12] Komal Sharma, Navneet Kaur, " Comparative Analysis of Various Edge Detection Techniques" , International Journal of Advanced Research in Computer Science and Software Engineering, IJARCSSE, ISSN: 2277 128X, Volume 3, Issue 12, December 2013.

[13] J. Selvakumar, A. Lakshmi, T. Arivoli, " Brain Tumor Segmentation and Its Area Calculation in Brain MR Images using K-Mean Clustering and Fuzzy C-Mean Algorithm" , IEEE-International Conference On Advances In Engineering, Science And Management, ICAESM, 2012.

[14] R. J. Ramteke1, Khachane Monali Y., " Automatic Medical Image Classification and Abnormality Detection Using K-Nearest Neighbour", International Journal of Advanced Computer Research,Volume2 Number-4 Issue-6 December-2012.

[15] Xiao Xuan, Qingmin Liao, Statistical Structure Analysis in MRI Brain Tumor Segmentation" ,IEEE International Conference on Image and Graphics, 2007.

[16] Mohd Fauzi Othman, Mohd Ariffanan, Mohd Basri, " Probabilistic Neural Network for Brain Tumor Classification" ,IEEE International Conference on Intelligent Systems, Modelling and Simulation,2011.

[17] Shweta Jain, "Brain Cancer Classification Using GLCM Based Feature Extraction in Artificial Neural Network" , International Journal of Computer Science \& Engineering Technology ,IJCSET, ISSN : 22293345 Vol. 4 No. 07 Jul 2013.

[18] Dina Aboul Dahab, Samy S. A. Ghoniemy, Gamal M. Selim, "Automated Brain Tumor Detection and Identification Using Image Processing and Probabilistic Neural Network Techniques" ,International Journal of Image Processing and Visual Communication, ISSN 2319-1724 : Volume (Online) 1, Issue 2 , October 2012.

[19] Walaa Hussein Ibrahim, Ahmed Abdel Rhman Ahmed Osman, Yusra Ibrahim Mohamed, "MRI Brain Image Classification Using Neural Networks" ,IEEE International Conference On Computing, Electrical and Electronics Engineering, ICCEEE,2013.

[20] Noramalina Abdullah, Lee Wee Chuen, Umi Kalthum Ngah Khairul Azman Ahmad, "Improvement of MRI Brain Classification using Principal Component Analysis" , IEEE International Conference on Control System, Computing and Engineering, 2011.

[21] Mehdi Jafari, Reza Shafaghi, "A Hybrid Approach for Automatic Tumor Detection of Brain MRI using Support Vector Machine and Genetic Algorithm", Global Journal of Science Engineering and Technology, Issue-3, 2012.

[22] V. Salai Selvam and S. Shenbagadevi, "Brain Tumor Detection using Scalp EEG with Modified WaveletICA and Multi Layer Feed Forward Neural Network" , Annual International Conference of the IEEE EMBS Boston, Massachusetts USA, August 30 - September 3, 2011.

[23] Amir Shahzad, Muhammad Sharif, Mudassar Raza, Khalid Hussai, " Enhanced Watershed Image Processing Segmentation ", Journal of Information \& Communication Technology, Vol. 2, No. 1, 2009. 